\title{
Potentilla pedata Willd. ex Hornem. (Rosaceae), novedad para la Península lbérica
}

\section{Pere Aymerich ${ }^{1} \&$ Llorenç Sáez ${ }^{2,3}$}

${ }^{1}$ C. Barcelona, 29. 08600 Berga, Barcelona.

2. Systematics and Evolution of Vascular Plants (UAB) - Associated Unit to CSIC. Dept. Biologia Animal, Vegetal i Ecologia, Facultat de Biociències, Universitat Autònoma de Barcelona, 08193 Bellaterra, Spain

${ }^{3}$ Societat d'Història Natural de les Illes Balears, c/ Margarida Xirgu 16, 07003 Palma de Mallorca, Balearic Islands, Spain.

\section{Correspondencia}

Pere Aymerich

e.mail: pere_aymerich@yahoo.es

Recibido: 2 diciembre 2020

Aceptado: 14 enero 2021

Publicado on-line: 25 enero 2021

Editado por: Marta Recio Criado

\section{Resumen}

Se documenta la presencia en los Pirineos de Potentilla pedata, sobre la base de criterios morfológicos ampliamente aceptados en los últimos años en la zona mediterránea. Esta especie presenta caracteres en parte intermedios entre los de $P$. recta y $P$. hirta.

Palabras clave: Potentilla sect. Recta, corología, Pirineos, España, Cataluña.

\section{Abstract}

Potentilla pedata Willd. ex Hornem. (Rosaceae), new for the Iberian Península

The presence of Potentilla pedata is reported in the Pyrenees, based on morphological criteria largely accepted the last years in the Mediterranean region. This species shows intermediate characters between $P$. recta and $P$. hirta.

Key words: Potentilla sect. Recta, chorology, Pyrenees, Spain, Catalonia.
Potentilla pedata Willd. ex Hornem., Hort. Bot. Hafn. 2: 477 (1815)

Neotipo: C (Tison \& Malécot, 2007: 9)

ESPAÑA, Lleida: Espot, Bosc de Vellendo, entre los núcleos de Estaís y Jou, 31TCG4615, 1370 m s.n.m, pasto xeromesófilo sobre esquistos carbonatados, en una vertiente de orientación SE, 29-VIII-2020, P. Aymerich (BCB) (Fig. 1).

En agosto de 2020 encontramos en una localidad de los Pirineos centrales una pequeña población de Potentilla L. sin duda referible a la sect. Recta (Th. Wolf) Juzepchuck, pero que mostraba caracteres que no se correspondían con los atribuidos a los dos taxones de este grupo $(P$. recta $\mathrm{L}$. y $P$. hirta $\mathrm{L}$.) reconocidos en esta zona por las floras regionales modernas (Bolòs \& Vigo, 1984; Guillén \& Rico, 1998; Bolòs et al., 2005). Estas plantas presentaban un aspecto general similar al de $P$. asturica Rothm., el tercer taxón de la sección actualmente aceptado en la Península lbérica y endémico de las montañas de su cuadrante noroeste (Guillén \& Rico, 1998), pero se separaban con claridad de éste por varios caracteres. En cambio, se acomodaban bien a los caracteres generales que las floras francesas recientes (Tison \& Foucault, 2014; Tison et al., 2014) atribuyen a $P$. pedata Willd. ex Hornem., taxón hasta el momento no citado en el ámbito ibérico.

La taxonomía de Potentilla sect. Recta es compleja y, en el ámbito europeo, se ha interpretado de formas diversas y cambiantes con el tiempo. Dos especies han tenido una aceptación generalizada desde antiguo, $P$. hirta y $P$. recta, pero siempre se ha reconocido la existencia de poblaciones con caracteres intermedios, que no se podían atribuir de forma clara a ninguna de estas dos especies. Esta incertidumbre taxonómica se debe a que los caracteres cualitativos claros para separar taxones son escasos o inexistentes, por lo que en general se ha recurrido al uso de caracteres cuantitativos. En la práctica, gran parte de la dificultad taxonómica de Potentilla sect. Recta deriva del hecho de que $P$. recta y $P$. pedata representan complejos poliploides (Delgado et al., 2000), lo que explica su heterogeneidad morfológica y la frecuencia con que se podrían confundir, mientras que $P$. hirta y $P$. asturica Rothm. son diploides. Esta última especie es un endemismo ibérico de este grupo, que históricamente ha sido tratado también como subespecie de $P$. recta o de $P$. hirta (Guillén \& Rico, 1998).

A escala europea amplia, el "taxón intermedio" más controvertido ha sido $P$. pedata, que fue en general aceptado en el siglo XIX y en la primera mitad del $X X$, pero después se tendió a integrar en $P$. recta o en $P$. hirta, con la excepción relativa del este de Europa, donde $P$. pedata se ha reconocido como especie con relativa continuidad (e.g. TofanDorofeev, 2016). En tiempos modernos y en trabajos de ámbito continental, $P$. pedata fue incluida en una $P$. recta s.l. por Ball et al. (1968). Con posterioridad, fue aceptada como especie, supuestamente hibridógena entre $P$. hirta y $P$. recta y presente en el sur de Europa por Soják (1993). También es aceptada 
como especie en las síntesis corológicas de Kurtto et al. (1994) y Kurtto (2009), en las que se le atribuye una distribución que abarca el sur de Europa desde Francia hasta Ucrania, Turquía y el Cáucaso.

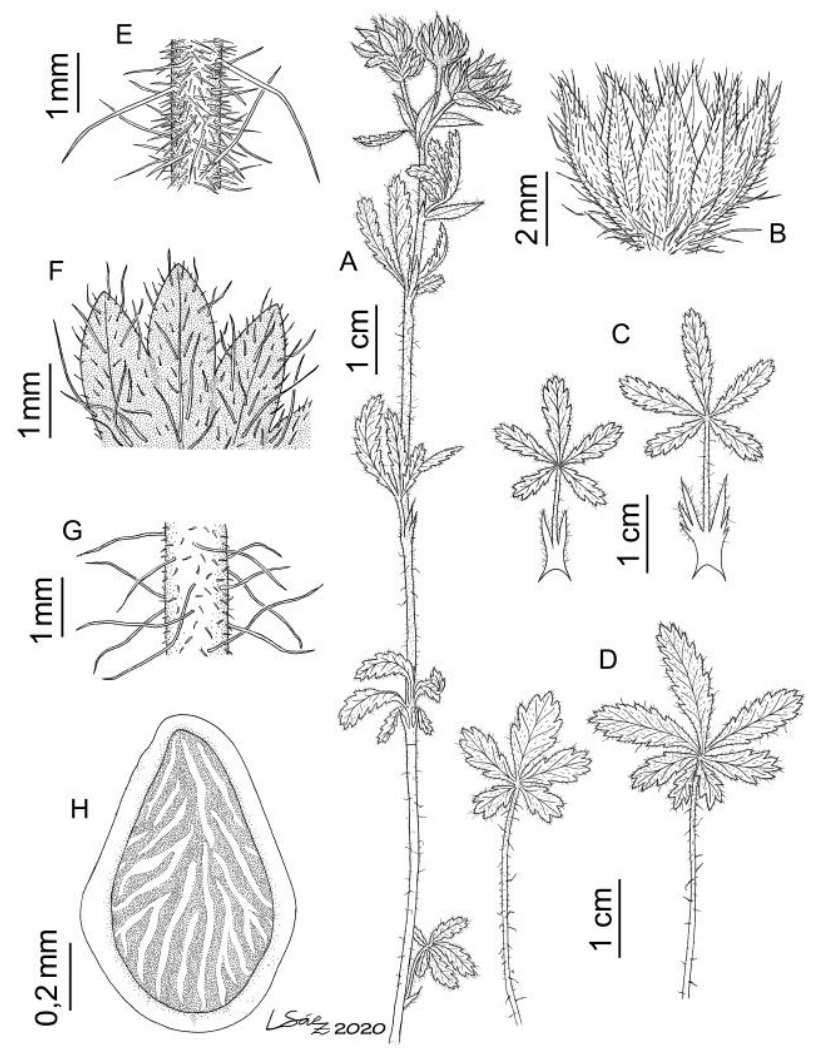

Figura 1. Potentilla pedata (Pallars Sobirà; Espot). A: hábito; B: flor tras la antesis; C: hojas caulinares; D: hojas basales; E: detalle de la pelosidad del tallo florífero (zona superior); F: detalle de la pelosidad del haz de una hoja caulinar media; G: detalle de la pelosidad del tallo florífero (zona media); $\mathrm{H}$ : aquenio.

Figure 1. Potentilla pedata (Pallars Sobirà; Espot). A: habit; $B$ : flower after anthesis; $C$ : cauline leaves; $D$ : basal leaves; $E$ : hairiness of the stem (upper zone); F: hairiness of middle cauline leaf; $G$ : hairiness of the stem (middle zone); H: fruit.

En el Mediterráneo central y occidental, después de una aceptación inicial de la presencia de $P$. pedata (Coste, 1893), las floras regionales publicadas en el siglo XX en general asignaron las plantas que presentaban caracteres compatibles con este taxón a $P$. hirta o a $P$. recta. Solamente desde la reinterpretación propuesta por Tison \& Malécot (2007) se ha recuperado la aceptación de la presencia en esta zona de $P$. pedata, primero en Francia y después en Italia. La reinterpretación ha resultado espectacular en Italia, donde de no aceptarse P. pedata (Conti et al., 2005; Pignatti, 2017), se ha pasado a considerar que está ampliamente extendida en casi todo el territorio, isla de Cerdeña incluida (e.g. Lazzeri et al., 2015 y a asumir que corresponden a esta especie la mayor parte -0 quizá todas- las citas previas de P. hirta, así como parte de las de $P$. recta.
Hasta ahora, esta reinterpretación moderna de $P$. pedata no había influido en el conocimiento de la flora ibérica, si bien existen dos pliegos antiguos de las Cordilleras Béticas (Sierra de Segura MA55447; Sierra de la Cabrilla P04176177) que fueron inicialmente asignados a $P$. pedata y años después a $P$. recta, y por lo menos el primero presenta gran parte de los caracteres compatibles con la reinterpretación reciente de $P$. pedata.

En esta nota se aportan por primera vez datos sobre la presencia de $P$. pedata, según la interpretación de Tison \& Malécot (2007), en la vertiente meridional de los Pirineos, lo que supone una novedad para la flora ibérica. La identificación de estas plantas se ha basado en los criterios morfológicos utilizados por Tison \& Malécot (2007) para separar esta especie de $P$. recta y de $P$. hirta. La delimitación de estas dos últimas especies en este trabajo no coincide completamente con la utilizada en el ámbito ibérico por Guillén \& Rico (1997) y Rico et al. (2003). Según los criterios de Tison \& Malécot (2007) parte de la variabilidad asignada a $P$. recta o $P$. hirta en los trabajos ibéricos -en especial la variabilidad extrema- podría entrar en la delimitación que el primer artículo atribuye a $P$. pedata.

A continuación se exponen los caracteres fundamentales por los que se ha asignado el material pirenaico a $P$. pedata. La asignación taxonómica se ha basado en el criterio de Tison \& Malécot (2007), comentando sucintamente la variabilidad expuesta para algunos taxones en trabajos de ámbito ibérico (Guillén \& Rico, 1998; Rico et al., 2003).

Porte general: La muestra ibérica atribuida a $P$. pedata presenta unas dimensiones y coloración del tallo (parcialment rojizo) concordantes con la variabilidad de esta especie según Tison \& Malécot (2007), aunque se trata de caracteres de valor diagnóstico discutible. El porte es erecto, también dentro de la variabilidad descrita por los autores citados, que atribuyen a $P$. pedata un porte variable, mientras que $P$. recta sería siempre erecta y $P$. hirta ascendente, caracteres que en general son también aceptados por otros autores. La morfología general de nuestras plantas presenta ciertas semejanzas con la de $P$. asturica, si bien esta especie presenta en general tallos ascendentes y sólo excepcionalmente erectos (Guillén \& Rico, 1998).

Hojas: El material ibérico asignado a $P$. pedata presenta el segmento central de las hojas caulinares medias entero en el 1/3 basal como máximo y con 15-17 lóbulos, dentro de la variabilidad de esta especie según Tison \& Malécot (2007) pero sin encajar con $P$. recta (17-31 dientes, presentes también en la mitad inferior) ni $P$. hirta (5-7 dientes, solo en la mitad apical). Nuestras muestras presentaban en vivo segmentos levemente plegados en sentido longitudinal, carácter poco visible en material de herbario y que según Tison \& Malécot (2007) puede darse en $P$. pedata pero no en $P$. 
recta, mientras que $P$. hirta tendría siempre hojas claramente plegadas.

Estípulas: En nuestro material son inciso-dentadas o enteras, variabilidad que concuerda con lo atribuido por Tison \& Malécot (2007) para $P$. pedata, mientras que $P$. recta tendría estípulas divididas y $P$. hirta enteras. La interpretación de los caracteres de las estípulas de Guillén \& Rico (1998) difiere de lo antes indicado, pues estos autores consideran que $P$. recta puede tener estípulas dentadas o enteras, mientras que $P$. hirta las tendría generalmente enteras pero en algún caso con dientes. La información proporcionada por Rico et al. (2003) es intermedia, ya que se indica que cerca de un tercio de los ejemplares estudiados de $P$. recta presenta estípulas dentadas, pero $P$. hirta siempre las presentaría enteras.

Indumento de pelos eglandulíferos cortos: Nuestra muestra presenta diversos tipos de pelos, eglandulíferos y glandulíferos, y largos o cortos (máximo $3,5 \mathrm{~mm}$ ). Entre los pelos eglandulíferos, parece que únicamente los cortos tendrían valor diagnóstico, aunque Tison \& Malécot (2007) no precisan el rango de longitud para el que un pelo se considera "corto". Si se consideran sólo los pelos de hasta $0,3 \mathrm{~mm}$ de longitud -pelos "cortos" según Rico et al. (2003)éstos son muy abundantes en el tercio superior de la planta, decreciendo progresivamente su densidad hacia la base, donde son muy dispersos o nulos. Este carácter concuerda en general con lo atribuido por Tison \& Malécot (2007) a $P$. pedata, especie que tendría pocos o bastantes pelos largos, abundantes pelos cortos en la parte superior del tallo y sin pelos cortos en la base.

La densidad de pelos cortos permite separar $P$. pedata de $P$. asturica, ya que en ésta los pelos son abundantes en la mitad basal del tallo (Rico et al., 2003). En cuanto a la anchura basal de los pelos largos, carácter no utilizado por Tison \& Malécot (2007) pero que Rico et al. (2003) consideran de valor diagnóstico en Potentilla sect. Recta, en nuestras plantas es en general inferior a 25 micras, mientras que los que superan dicho valor (alcanzando hasta 38 micras en su base) son más bien escasos. Estos valores se sitúan entre los de $P$. asturica y $P$. hirta (medias respectivas de 15,0 y 33,5 micras) según Rico et al. (2003) y se alejan notablemente de los de $P$ recta (media de 42,6 micras), que es la única especie hasta ahora confirmada en la zona pirenaica donde encontramos $P$. pedata.

Indumento de pelos glandulíferos: En nuestra muestra, los pelos glandulíferos son relativamente cortos, hasta de $1,7(2) \mathrm{mm}$ de longitud, y se encuentran muy dispersos en las ramas de la inflorescencia y en las hojas caulinares. Según Tison \& Malécot (2007), en $P$. pedata la presencia de pelos glandulíferos en las partes superiores es entre nula y débil, por lo que la muestra pirenaica entraría dentro de su varia- bilidad. Según estos autores, en general están presentes y a menudo son abundantes en $P$. recta, mientras que no existirían en $P$. hirta.

De acuerdo con las descripciones de plantas ibéricas, la presencia de pelos glandulíferos pluricelulares está documentada en $P$. recta (Guillén \& Rico, 1998), en la que este tipo de indumento es común en la parte superior de la planta, hojas (sobre todo las caulinares) y especialmente en la inflorescencia. Por el contrario, ni $P$. asturica ni $P$. hirta presentarían pelos glandulíferos.

Se han analizado también las anteras y los aquenios, las medidas de los cuales están en el límite o fuera de lo que Tison \& Malécot (2007) consideran propio de $P$. pedata, pero la interpretación de estos resultados es incierta, ya que estos autores no precisan el método para obtener estos valores.

En conjunto, con la suma de los caracteres analizados en las plantas halladas en los Pirineos catalanes, consideramos que éstas se pueden adscribir a $P$. pedata de acuerdo con el criterio de Tison \& Malécot (2007). Se aprecian algunas discordancias menores con los rangos de variabilidad atribuidos a $P$. pedata por estos autores (tamaño de las anteras y del ala de los aquenios, presencia de algún pelo corto en la mitad inferior del tallo), que podrían ser debidas a que el trabajo de Tison \& Malécot (2007) se basa en materiales franceses y quizá no refleja suficientemente la variabilidad de $P$. pedata, así como también a la imprecisión de los criterios utilizados para registrar algunos caracteres (definición de parte basal del tallo o anchura del ala del aquenio; estado de las flores en que hay que medir las anteras).

La localidad pirenaica aportada es la primera de $P$. pedata en la Península lbérica según esta interpretación reciente de la especie. Representa también la localidad más occidental confirmada, pues las citas más próximas reflejadas en la bibliografía corresponden al área de las Causses, en el borde meridional del Macizo Central francés, donde esta especie ya se citó antiguamente (Coste, 1893) y se ha confirmado en tiempos recientes (Tison et al., 2014). Existe también una cita informal, pero aparentemente bastante fiable, en la vertiente norte del macizo de Corbières, al sur del Languedoc (AFP, 2020: dato original del Conservatoire Botanique National Méditerranéen), zona que se sitúa a una distancia similar de las Causses y de la nueva localidad pirenaica.

Se desconoce si $P$. pedata se encuentra en otras zonas ibéricas, pero se considera probable, ya que parte de la variabilidad reflejada por Guillén \& Rico (1998) para $P$. recta y $P$. hirta podría corresponder a poblaciones actualmente referibles a $P$. pedata. En cualquier caso, consideramos que la taxonomía de Potentilla sect. Recta en el Mediterráneo occidental requiere estudios más detallados, ya que tanto la propuesta de Tison \& Malécot (2007) como otras previas presentan algunas incoherencias. 
A continuación se propone una clave dicotómica para las especies de Potentilla sect. Recta en la Península Ibérica:

1. Hojas caulinares medias con el segmento medio con 3-7 dientes repartidos en menos de la mitad distal de su longitud........................................ hirta - Hojas caulinares medias con el segmento medio con (7)9-33 dientes repartidos en más de la mitad distal de su longitud......

2. Hojas caulinares medias con el segmento medio con 17-33 dientes; estípulas medias incisodentadas; pelos glandulíferos frecuentes, al menos en la inflorescencia; carpelos, en general, más de 80 por flor. P. recta - Hojas caulinares medias con el segmento medio con 7-17 dientes; estípulas medias inciso-dentadas o enteras; pelos glandulíferos nulos 0 muy dispersos; carpelos, en general, menos de 80 por flor. .3 3. Pelos eglandulíferos cortos en la zona basal del tallo de frecuentes a abundantes; estípulas enteras (excepcionalmente 2-fidas); tallos casi siempre ascendentes............................................ P. asturica - Pelos eglandulíferos cortos en la zona basal del tallo nulos o muy escasos; estípulas de enteras a 3fidas; tallos ascendentes o erectos P. pedata

\section{Agradecimientos}

Deseamos agradecer al personal del herbario BC, y en especial a Neus Nualart, su ayuda (localización de materiales) en nuestra visita en este herbario. Josep Vicens (herbario BCN) proporcionó información útil sobre $P$. recta. Agradecemos, asimismo, a Leopoldo Medina y Alberto Herrero (herbario MA) habernos proporcionado imágenes de algunos materiales de herbario. Finalmente, queremos agradecer a Ignasi Soriano (Univ. de Barcelona) su ayuda en la obtención de bibliografía especializada y a Enrique Rico (Univ. de Salamanca) las críticas y consejos que han ayudado a mejorar esta aportación.

\section{Bibliografía}

AFP (2020). Atlas de la Flora de los Pirineos en línea.www.florapyrenaea.com/florapyrenaea/inde x.jsp. Consulta realizada en 23-11-2020.

Ball, P.W., Pawlowski, B. \& Walters, S.M. (1968). Potentilla L. In: T.G. Tutin, V.H. Heywood, N.A. Burges, D.M. Moore, D.H Valentine, S.D. Walters \& D.A. Webb (eds.). Flora Euopaea. Vol. 2: Rosaceae to Umbelliferae. (pp. 36-47).
Cambridge, UK: Cambridge, University Press.

Bolòs, O. \& Vigo, J. (1984). Flora dels Països Catalans. Vol. 1. Barcelona: Ed. Barcino.

Bolòs, O., Vigo, J., Masalles, R.M. \& Ninot, J.M. (2005). Flora manual dels Països Catalans. Barcelona: Ed. Pòrtic.

Coste, H. (1893). Florule Du Larzac, Du Causse Noir Et Du Causse De Saint-Affrique, Bulletin de la Société Botanique de France, 40, 10, XCI-CXL.

Delgado, L. \& Rico, E. (2000). Karyosystematic study of Potentilla L. subgen. Potentilla (Rosaceae) in the Iberian Peninsula. Botanical Journal of the Linnean Society, 132(3), 263-280. https://doi.org/10.1111/j.1095-8339.2000.tb01531.x

Guillén, A. \& Rico E. (1998). Potentilla L. In: F. Muñoz Garmendia \& C. Navarro (eds.). Flora iberica, Vol. 6. Madrid: Real Jardín Botánico-C.S.I.C.

Kurtto, A., Lampinen, R. \& Junnikka L. (2004). Atlas Flora Europaeae: Distribution of Vascular Plants in Europe. 13: Rosaceae (Spiraea to Fragaria, excl. Rubus). Helsinki: Comittee for Mapping the Flora of Europe and Socetas Biologica Fennica Vanamo.

Kurtto, A. (2009). Rosaceae (pro parte majore). Euro+Med Plantbase - the information resource for Euro-Mediterranean plant diversity. http://ww2.bgbm.org/EuroPlusMed. Consulta realizada en 23-11-2020.

Lazzeri, V., Sanmartino, F., Campus, G., Caredda, A., Mascia, F., Mazzoncini, V., Testa, N. \& Gestri, G. (2015). Note floristiche Tosco-Sarde II: Novità regionali e local e considerazioni tassonomiche per le regione Sardegna e Toscana. Annali Museo Civico Rovereto, 30, 331-368.

Pignatti, S. (2017). Flora d'Italia. Vol. 2. Milano: Edagricole.

Rico, E., Martínez-Ortega, M., Delgado, L., Baez, A. \& Martínez, A. (2003). A multivariate morphometric of the Iberian representatives of Potentilla sect. Recta (Rosaceae). Folia Geobotanica, 38, 35-48. https://doi.org/10.1007/BF02803126

Soják, J. (1993). Taxonomische Bemerkungen zu einigen mediterranen Potentilla-Sippen. Preslia, 65, 117-130.

Tison J.-M. \& Malécot V. (2007). Potentilla pedata Willd. ex Hornem. (Rosaceae), espèce francaise méconnue. Le Monde des Plantes, 493, 5-16.

Tison, J.M. \& Foucault, B. (2014) (coords.). Flore Gallica. Flore de France. Mèze, FR: Ed. Biotope.

Tison, J.M., Jauzein, P. \& Michaud, H. (2014). Flore de la France méditerranéenne continentale. Conservatoire Botanique National Méditerranéen de Porquerolles. Turriers, FR: Naturalia Publications.

Tofan-Dorofeev, E. (2016). The genus Potentilla L. (Rosaceae Juss.) in the Flora of Bessarabia. Revista Botanica, 1, 66-72. 